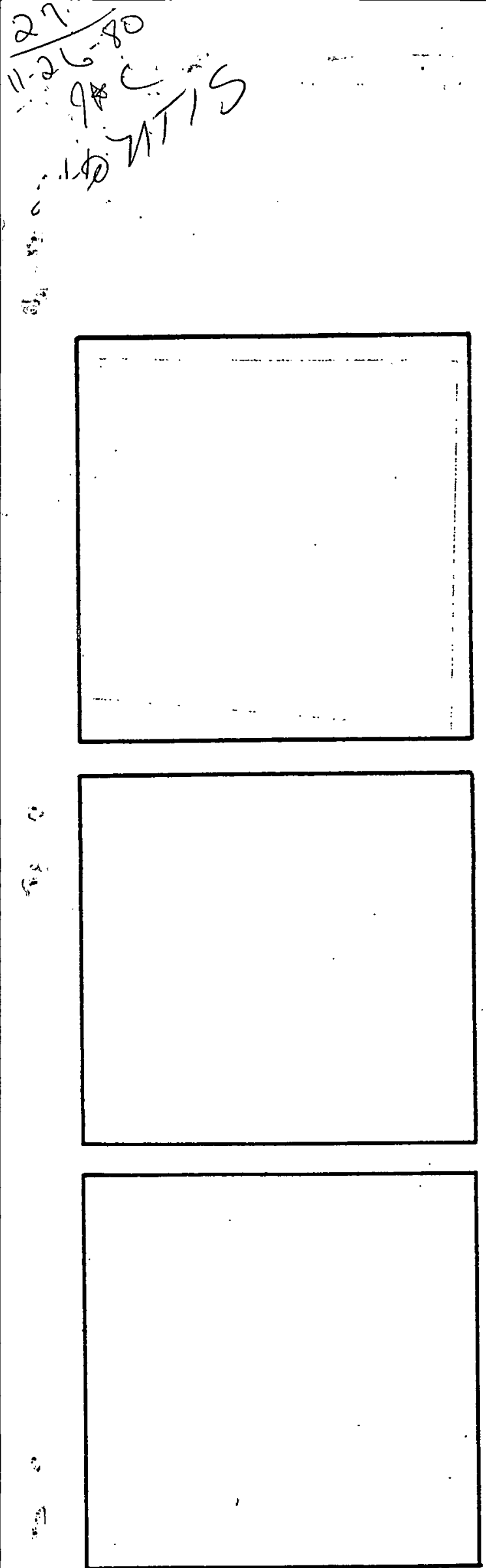

BDX-613-2409

\title{
A Modified Thin Film Processing Sequence
}

By C. R. Baxter

Published June 1980

Topical Report

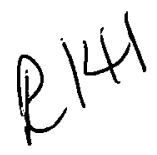

Prepared for the United States Department of Energy Under Contract Number DE-AC04-76-DP00613.

\section{Bendix Kansas City Division}




\section{DISCLAIMER}

This report was prepared as an account of work sponsored by an agency of the United States Government. Neither the United States Government nor any agency Thereof, nor any of their employees, makes any warranty, express or implied, or assumes any legal liability or responsibility for the accuracy, completeness, or usefulness of any information, apparatus, product, or process disclosed, or represents that its use would not infringe privately owned rights. Reference herein to any specific commercial product, process, or service by trade name, trademark, manufacturer, or otherwise does not necessarily constitute or imply its endorsement, recommendation, or favoring by the United States Government or any agency thereof. The views and opinions of authors expressed herein do not necessarily state or reflect those of the United States Government or any agency thereof. 


\section{DISCLAIMER}

Portions of this document may be illegible in electronic image products. Images are produced from the best available original document. 
This report was prepared as an account of work sponsored by the United States Government. Neither the United States nor the United States Department of Energy, nor any of their employees, nor any of their contractors, subcontractors, or their employees, makes any warranty, express or implied, or assumes any legal liability or responsibility for the accuracy, completeness or usefulness of any information, apparatus, product or process disclosed, or represents that its use would not infringe privately owned rights.

Printed in the United States of America

Available From the National Technical Information Service, U.S. Department of Commerce, 5285 Port Royal Road, Springfield, Virginia 22161.

Price: Microfiche $\$ 3.00$

Paper Copy $\$ 4.00$ 


\section{A MODIFIED THIN FILM PROCESSING SEQUENCE}

By C. R. Baxter

Published June 1980

Topical Report

C. R. Baxter, Project Leader

Project Team:

R. W. Pierce

W. A. Piper

J. H. Pusch

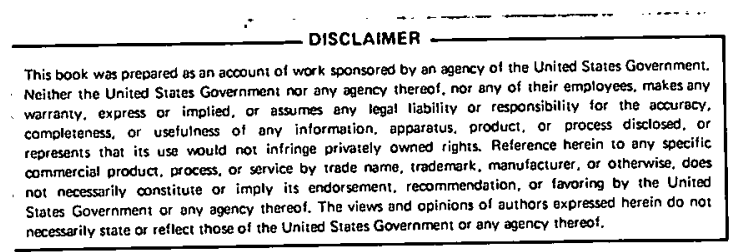


A MODIFIED THIN FILM PROCESSING SEQUENCE

BDX-613-2409, Topical Report, Published June 1980

Prepared by C. R. Baxter

A modified thin film processing sequence in which tantalum nitride $\left(\mathrm{TA}_{2} \mathrm{~N}\right)$ resistors are stabilized prior to chromium/gold ( $\left.\mathrm{Cr} / \mathrm{Au}\right)$ evaporation was investigated, and the effects of subsequent processing on unstabilized $\mathrm{Cr} / \mathrm{Au}$ films were determined. Thin films evaporated using the modified process yielded results similar to those of films evaporated using the standard processing techniques. Work on the modified process was discontinued because of some metallization adhesion failures at the $\mathrm{Cr} / \mathrm{Au}-\mathrm{to}-\mathrm{Ta}_{2} \mathrm{~N}$ interface, higher contact resistance, and the additional steps required in processing.

TWL/TR3

This report was prepared as an account of work sponsored by the United States Government. Neither. the United States, nor the United States Department of Energy, nor any of their employees. nor any of their contractors. subcontractors, or their employees. makes any warranty, expressed or implied or assumes any legal liability or responsibility for the accuracy. completeness or usefulness of any information, apparatus, product, or process disclosed, or represents that its use would not infringe privately owned rights.
The Bendix Corporation Kansas City Division P. O. Box 1159 Kansas City. Missouri 64141

A prime contractor with the United States Department of Energy under Contract Number DE-AC04-76-DPO0613 


\section{CONTENTS}

Section
SUMMARY. . . . . . . . . . . . . . . . . . . . . . .


TABLES

Number

Page

1

Process Flow Sequence. . . . . . . . . . .

Summary of Modified Process Evaluation . . .

Temperature Coefficient of Resistance for Standard Process Films. . . . . . . . .

Temperature Coefficient of Resistance for Modified Process Films . . . . . . . . .

Life Stability of Standard Process Films . .

Life Stability of Modified Process Films . . .

Failure Mode Definitions for Lead Frame Bonding.

Accelerated Aging ( 4 Hours at $200^{\circ} \mathrm{C}$ ) Versus Thermocompression Bondability for Standard and Modified Process Films . . . . . . . .

Thermocompression Bondability of Back Side Metallization. . . . . . . . . . . . .

Thermocompression Bondability of Front Side "J" Circuits, Modified Process . . . . . .

Age-Bond-Pull Results for Standard and Modified Process Films . . . . . . . . .

Bond-Age-Pull Results for Standard and Modified Process Films . . . . . . . . 20 


\section{SUMMARY}

A modified thin film processing sequence was investigated as a means of minimizing metallization adhesion problems between the evaporated chromium/gold $(\mathrm{Cr} / \mathrm{Au})$ conductive layer and the sputtered tantalum nitride $\left(\mathrm{Ta}_{2} \mathrm{~N}\right)$ resistive layer of thin film hybrid microcircuits. During normal production processing ceramic substrates are sputtered with $\mathrm{Ta}_{2} \mathrm{~N}$ (resistive layer), evaporated with $\mathrm{Cr} / \mathrm{Au}$ (conductive layer), and then patterned using photolithographic techniques. The thin film resistors are then stabilized in air for 2 hours at $300^{\circ} \mathrm{C}$. In the modified processing sequence, a $100 \mathrm{~nm}$ chromium film is evaporated over the sputtered tantalum nitride layer. The desired resistor pattern is then photodefined with chromium left covering the underlying tantalum nitride everywhere conductor contact is required. The resistors are then stabilized in air for 2 hours at $300^{\circ} \mathrm{C}$, the protective chromium layer is etched away, and the final $\mathrm{Cr} / \mathrm{Au}$ film is deposited and patterned.

Films were deposited, using both the standard and the modified processes, and tested. Emphasis was given to the effect of omitting the protective chromium layer and to the effects of subsequent processing on unstabilized $\mathrm{Cr} / \mathrm{Au}$ films. Temperature coefficient of resistance (TCR), resistor life stability, via resistance, bondability, and solderability were compared for both standard and modified process films. TCR values for modified process films were more negative than standard process films, but were well within specification limits. Life stability data was also within specification limits for both standard and modified process films. Omission of the $100 \mathrm{~nm}$ protective layer resulted in life stability data that was considerably outside specification limits. Via resistance was excellent for both types of processes. Lead frame bondability results were similar for both types of processing, but some metallization adhesion failures were observed on modified process samples. There were no problems encountered in soldering to modified process films when using 50-50 $\mathrm{Pb}$-In solder.

Results of this investigation showed the modified thin film process would not offer any significant advantages over the present production process. The modified sequence requires additional processing during both evaporation and photolithography. Based on resulsts obtained at both Bendix Kansas City and Sandia National Laboratories, Albuquerque, the modified thin film processing sequence was also found to be sensitive to metallization adhesion failures and to have a contact resistance 2 to 3 times higher than the standard process. Consequently, it was decided to discontinue efforts on the modified process as a solution to metallization adhesion problems observed in manufacturing processes. 


\section{DISCUSSION}

\section{SCOPE AND PURPOSE}

The purpose of this work was to determine the feasibility of a modified tantalum nitride-chromium/gold $\left(\mathrm{Ta}_{2} \mathrm{~N}-\mathrm{Cr} / \mathrm{Au}\right)$ deposition sequence in which the tantalum nitride resistors are stabilized prior to chromium/gold deposition. A past history of adhesion. failures of the Cr/Au conductor metallization encountered in production with both $3000 \mathrm{~nm}$ and $6000 \mathrm{~nm}$ gold films prompted the need for a better understanding of the mechanisms leading to the degradation of film adhesion. The voiding in the chromium layer, which occurs when chromium diffuses through the gold during resistor stabilization, is considered a likely contributor to film adhesion failures. A modified tantalum nitride processing sequence was proposed by Sandia National Laboratories, Albuquerque (SNLA) to eliminate the chromium diffusion during resistor stabilization. The modified process was evaluated at Bendix Kansas City.

\section{PRIOR WORK}

Tests conducted at SNLA showed that, during resistor stabilization for 2 hours at $300^{\circ} \mathrm{C}$, voids form in the chromium "glue layer" between the tantalum nitride resistive layer and gold conductor layer. This void formation, a natural consequence of the diffusion mechanism of chromium through gold during resistor stabilization, was identified as a likely contributor to metallization adhesion failures experienced in thin film production. A modified process sequence was suggested by SNLA to perform stabilization prior to $\mathrm{Cr} / \mathrm{Au}$ deposition, thus eliminating chromium diffusion and voiding during stabilization as well as the corrosive prebond cericammonium-nitrate (CAN) etch, which is another likely contributor to film adhesion failures.

In the initial evaluation of the modified thin film processing sequence at SNLA, good thermocompression bondability results were obtained for lead frames, wires, and ribbons before and after films were aged at $200^{\circ} \mathrm{C}$ for 150 hours. SNLA evaluated contact resistance, resistor temperature coefficient of resistance (TCR), and life stability. Contact resistance for the modified process was three to four times higher than for the standard process. Average resistor life stability was greater than the 0.2 percent specification limit and individual resistor changes were as high as 3.2 percent. Resistor TCR for the modified process ranged from -100 to $-130 \mathrm{ppm} /{ }^{\circ} \mathrm{C}$. Specification limits are -60 to $-120 \mathrm{ppm} /{ }^{\circ} \mathrm{C}$........ Lead frame bondability studies on unaged thin film networks (TFNs), TFNs 
aged at $200^{\circ} \mathrm{C}$ for 4 hours, and TFNs aged at $250^{\circ} \mathrm{C}$ for 1 hour were conducted at SNLA. Type $\mathrm{M}\left(\mathrm{Cr} / \mathrm{Au}-\mathrm{to}-\mathrm{Ta}_{2} \mathrm{~N}\right)$ failures were obtained on TFNs processed according to both the modified and standard processes, and the modified process appeared to be less sensitive to subsequent processing than the standard process.

After SNLA had obtained promising results with the modified process, Bendix conducted a brief evaluation to identify processing steps where development would be required before consideration would be given to using the modified process in production. Five alumina substrates were processed according to the modified process sequence into TFNs. There were no observed metallization failures, but all of the TCR data and some of the resistor life stability data was outside specification limits. Based on previous data, a detailed laboratory evaluation of the modified process was conducted to establish process boundary conditions, to evaluate TCR and life stability, and to determine the effect of subsequent processing on unstabilized $\mathrm{Cr} / \mathrm{Au}$ films.

\section{ACTIVITY}

\section{Processing}

The standard production process for the manufacture of hybrid microcircuits at Bendix begins with 95 by 114 by $0.69 \mathrm{~mm}$ alumina substrates of 99.5 percent aluminum oxide. A layer of tantalum nitride is sputtered. on the substrates followed by vacuum evaporated layers of chromium and gold respectively. Typical film thickness are $50 \mathrm{~nm}$ of $\mathrm{Ta}_{2} \mathrm{~N}, 25 \mathrm{~nm}$ of chromium, and either $3000 \mathrm{~nm}$ or $6000 \mathrm{~nm}$ of gold. The $\mathrm{Ta}_{2} \mathrm{~N}$ is used for thin film resistors, and the $\mathrm{Cr} / \mathrm{Au}$ is used for conductors. The chromium serves as an adhesive layer between the tantalum nitride and the gold.

Photolithographic procedures are used to process substrates to the thin film network (TFN) level. A TFN is typically 25 by $25 \mathrm{~mm}$. Two different mask levels are required to produce the TFN pattern. The first mask level is for the conductor pattern and the second is for the resistor pattern. After the substrates have been photoprocessed, the resistors are thermally stabilized in air at $300^{\circ} \mathrm{C}$ for 2 hours. The large substrates are then laser scribed and separated into smaller TFNs. The resistors are trimmed to nominal value by means of an automated laser trimmer. During the resistor stabilization process, chromium diffuses to the gold surface and oxidizes, which decreases the thermocompression bondability of the substrates. Consequently, prior to component attachment and bonding operations, the chromium oxide layer is removed by a cerric-ammonium-nitrate (CAN) etch. 
In the modified processing sequence, a $100-\mathrm{nm}$ chromium film is evaporated over the sputtered $\mathrm{Ta}_{2} \mathrm{~N}$ layer. Photolithographic procedures are then used to define the desired resistor pattern with chromium left covering the underlying $\mathrm{Ta}_{2} \mathrm{~N}$ everywhere that conductor contact with the $\mathrm{Ta}_{2} \mathrm{~N}$ resistors is required. The purpose of the chromium film is to prevent oxidation of the $\mathrm{Ta}_{2} \mathrm{~N}$ and to eliminate any problems of contact resistance between the conductor and resistor films. The resistors are then stabilized in air for 2 hours at $300^{\circ} \mathrm{C}$, the protective chromium layer is etched away, and the final Cr/Au films are deposited and patterned. The early stabilization of the $\mathrm{Ta}_{2} \mathrm{~N}$ resistors eliminates void formation in the chromium layer as a result of chromium diffusion during stabilization and eliminates the need for the CAN etch step prior to bonding and component attachment. Processing steps for both the standard and modified processes are listed in Table 1.

A laboratory investigation of the modified $\mathrm{Ta}_{2} \mathrm{~N}$ process was started. The processing summary for this evaluation is summarized in Table 2. Fifteen ceramic substrates, 95 by 114 by $0.69 \mathrm{~mm}$, were processed into thin film networks measuring 25 by 25 by $0.69 \mathrm{~mm}$. Twelve ceramic substrates were processed using the modified sequence, and three of the larger substrates were processed using the standard process for control. Production equipment (dc sputtering, E-beam evaporation, and photolithography) were used in TFN processing. The primary objectives of this evaluation were to evaluate photolithographic processing techniques, to determine the effects of omitting the protective chromium layer, to check the repeatability of previous Bendix results using the modified process, and to determine the effects of subsequent processing on unstabilized $\mathrm{Cr} / \mathrm{Au}$ films. All of the films in this evaluation had a $6000 \mathrm{~nm}$ thickness of evaporated gold.

\section{Temperature Coefficient of Resistance Testing}

Temperature coefficient of resistance (TCR) was measured on five resistors at $-50,100$, and $150^{\circ} \mathrm{C}$ for films processed by both the standard and modified sequences. These 15 values were then averaged and the results are shown in Tables 3 and 4 . The average TCR for modified process films was $-105 \mathrm{ppm} /{ }^{\circ} \mathrm{C}$, and the control films had an average TCR of $-99.5 \mathrm{ppm} /{ }^{\circ} \mathrm{C}$. Specification limits for TCR are $-90 \pm 30 \mathrm{ppm} /{ }^{\circ} \mathrm{C}$.

\section{Resistor Life Stability Testing}

Life stability of the thin film resistors was obtained by measuring the resistance of each of five rresistors on each TFN, subjecting the TFNs to $150^{\circ} \mathrm{C}$ for 200 hours, remeasuring the resistors, and computing the average percent deviation for each TFN. Tables 5 and 6 show the average percent deviation in resistance after 
Table 1. Process Flow Sequence

\begin{tabular}{|c|c|c|}
\hline $\begin{array}{l}\text { Process } \\
\text { Number }\end{array}$ & $\begin{array}{l}\text { Standard } \\
\text { Process }\end{array}$ & $\begin{array}{l}\text { Modified } \\
\text { Process }\end{array}$ \\
\hline 1 & Substrate Cleaning & Substrate Cleaning \\
\hline 2 & Sputter $\mathrm{Ta}_{2} \mathrm{~N}$ & Sputter $\mathrm{Ta}_{2} \mathrm{~N}$ \\
\hline 3 & $\begin{array}{l}\text { Evaporate } 25 \mathrm{~nm} \mathrm{Cr} \\
\text { and } 3 \text { or } 6 \mu \mathrm{m} \mathrm{Au}\end{array}$ & Evaporate $100 \mathrm{~nm} \mathrm{Cr}$ \\
\hline 4 & $\begin{array}{l}\text { Photodefine Conductor- } \\
\text { Resistor Pattern }\end{array}$ & $\begin{array}{l}\text { Photodefine Conductor- } \\
\text { Resistor Pattern }\end{array}$ \\
\hline 5 & $\begin{array}{l}\text { Stabilize Resistors } \\
2 \text { Hours at } 300^{\circ} \mathrm{C}\end{array}$ & $\begin{array}{l}\text { Stabilize Resistors } 2 \text { Hours } \\
\text { at } 300^{\circ} \mathrm{C}\end{array}$ \\
\hline 6 & Laser Scribe and Break & Etch Remaining Cr Film \\
\hline 7 & CAN Etch & Predeposition Cleaning \\
\hline 8 & TC Clean & $\begin{array}{l}\text { Evaporate } 25 \mathrm{~nm} \mathrm{Cr} \text { and } 3 \\
\text { or } 6 \mu \mathrm{m} \mathrm{Au}\end{array}$ \\
\hline 9 & & Photodefine Conductor Pattern \\
\hline 10 & & Laser Scribe and Break \\
\hline 11 & & TC Clean \\
\hline
\end{tabular}

200 hours at $150^{\circ} \mathrm{C}$ for both standard and modified process films: Specifications state that the percentage change shall not exceed 0.2 percent. Table 6 shows that TFNs 031,032 , and 033 had life stability values which were considerably outside specification limits. These TFNs were all from the same size substrate, which was processed by the modified sequence, but did not receive the $100 \mathrm{~nm}$ protective chromium layer.

\section{Bondability Testing}

Thermocompression bonding was used to evaluate film adhesion and to assure reliable bond junctions. Thermocompression (TC) bonding is used for the attachment of both components and input-output leads to the hybrid circuits. In TC bonding, the bond is formed by inducing a suitable amount of material flow in one or both members of the bond by the application of pressure and heat so that adhesion takes place in the absence of a liquid phase. Sample circuits are removed at selected points in the processing sequence and subjected to a bondability test. The product is accepted or rejected on the basis of this test. 
Table 2. Summary of Modified Process Evaluation

\begin{tabular}{|c|c|c|c|c|c|c|c|c|c|c|}
\hline \multirow{2}{*}{$\begin{array}{l}\text { Serial } \\
\text { Number }\end{array}$} & \multicolumn{4}{|c|}{ Photolith I } & \multirow{2}{*}{$\begin{array}{l}\text { Early } \\
\text { Stabili- } \\
\text { zation }\end{array}$} & \multicolumn{4}{|c|}{ Photolith II } & \multirow{2}{*}{$\begin{array}{l}\text { Normal } \\
\text { Stabili- } \\
\text { zation }\end{array}$} \\
\hline & $(100 \mathrm{~nm})$ & Type & $\mathrm{Ta}_{2} \mathrm{~N}$ & Type & & $\mathrm{Cr} / \mathrm{Au}$ & Type & $\mathrm{Ta}_{2} \mathrm{~N}$ & Type & \\
\hline \multicolumn{11}{|l|}{ Lot 1} \\
\hline $\begin{array}{l}01-09 \\
10-18 \\
19-27 \\
28-36 \\
37-45 t\end{array}$ & $\begin{array}{l}\text { Yes } \\
\text { No } \\
\text { Yes } \\
\text { No } \\
\text { No }\end{array}$ & $\begin{array}{l}\text { Dry } \\
\text { Dry }\end{array}$ & $\begin{array}{l}\text { Yes } \\
\text { No } \\
\text { Yes } \\
\text { No } \\
\text { No }\end{array}$ & $\begin{array}{l}\text { Wet } \\
\text { Wet }\end{array}$ & $\begin{array}{l}\text { Yes } \\
\text { Yes } \\
\text { Yes } \\
\text { Yes } \\
\text { No }\end{array}$ & $\begin{array}{l}\text { Yes } \\
\text { Yes } \\
\text { Yes } \\
\text { Yes } \\
\text { Yes }\end{array}$ & $\begin{array}{l}\text { Dry } \\
\text { Dry } \\
\text { Dry } \\
\text { Dry } \\
\text { Dry }\end{array}$ & $\begin{array}{l}\text { No } \\
\text { Yes } \\
\text { No } \\
\text { Yes } \\
\text { Yes }\end{array}$ & $\begin{array}{l}\text { Wet } \\
\text { Wet } \\
\text { Wet }\end{array}$ & $\begin{array}{l}\text { No } \\
\text { No } \\
\text { No } \\
\text { No } \\
\text { Yes }\end{array}$ \\
\hline \multicolumn{11}{|c|}{ Lot Number 2} \\
\hline $\begin{array}{l}46-54 \\
55-63 \\
64-72 \\
73-81 \\
82-90\end{array}$ & $\begin{array}{l}\text { Yes } \\
\text { Yes } \\
\text { Yes } \\
\text { Yes } \\
\text { No }\end{array}$ & $\begin{array}{l}\text { Dry } \\
\text { Dry } \\
\text { Wet } \\
\text { Wet }\end{array}$ & $\begin{array}{l}\text { Yes } \\
\text { Yes } \\
\text { Yes } \\
\text { Yes } \\
\text { No }\end{array}$ & $\begin{array}{l}\text { Wet } \\
\text { Wet } \\
\text { Wet } \\
\text { Wet }\end{array}$ & $\begin{array}{l}\text { Yes } \\
\text { Yes } \\
\text { Yes } \\
\text { Yes } \\
\text { No }\end{array}$ & $\begin{array}{l}\text { Yes } \\
\text { Yes } \\
\text { Yes } \\
\text { Yes } \\
\text { Yes }\end{array}$ & $\begin{array}{l}\text { Dry } \\
\text { Dry } \\
\text { Wet } \\
\text { Wet } \\
\text { Dry }\end{array}$ & $\begin{array}{l}\text { No } \\
\text { No } \\
\text { No } \\
\text { No } \\
\text { Yes }\end{array}$ & Wet & $\begin{array}{l}\text { No } \\
\text { No } \\
\text { No } \\
\text { No } \\
\text { Yes }\end{array}$ \\
\hline \multicolumn{11}{|c|}{ Lot Number 3} \\
\hline $\begin{array}{l}91-99 \\
100-108 \\
109-117 \\
118-126 \\
127-135 \%\end{array}$ & $\begin{array}{l}\text { Yes } \\
\text { Yes } \\
\text { Yes } \\
\text { Yes } \\
\text { No }\end{array}$ & $\begin{array}{l}\text { Dry } \\
\text { Dry } \\
\text { Wet } \\
\text { Wet }\end{array}$ & $\begin{array}{l}\text { Yes } \\
\text { Yes } \\
\text { Yes } \\
\text { Yes } \\
\text { No }\end{array}$ & $\begin{array}{l}\text { Wet } \\
\text { Wet } \\
\text { Wet } \\
\text { Wet }\end{array}$ & $\begin{array}{l}\text { Yes } \\
\text { Yes } \\
\text { Yes } \\
\text { Yes } \\
\text { No }\end{array}$ & $\begin{array}{l}\text { Yes } \\
\text { Yes } \\
\text { Yes } \\
\text { Yes } \\
\text { Yes }\end{array}$ & $\begin{array}{l}\text { Dry } \\
\text { Dry } \\
\text { Wet } \\
\text { Wet } \\
\text { Dry }\end{array}$ & $\begin{array}{l}\text { No } \\
\text { No } \\
\text { No } \\
\text { No } \\
\text { Yes }\end{array}$ & Wet & $\begin{array}{l}\text { No } \\
\text { No } \\
\text { No } \\
\text { No } \\
\text { Yes }\end{array}$ \\
\hline
\end{tabular}

*Control samples processed according to standard production process. 
Table 3. Temperature Coefficient of Resistance for Standard Process Films

\begin{tabular}{ll}
\hline $\begin{array}{l}\text { TFN } \\
\text { Number }\end{array}$ & $\begin{array}{l}\text { Average } \\
\text { TCR } \\
\left(\mathrm{ppm} /{ }^{\circ} \mathrm{C}\right)\end{array}$ \\
\hline 040 & -97.8 \\
041 & -97.6 \\
042 & -100.2 \\
085 & -98.1 \\
086 & -96.6 \\
087 & -97.6 \\
130 & -102.5 \\
131 & -101.1 \\
132 & -103.8 \\
\hline
\end{tabular}

Table 4. Temperature Coefficient of Resistance for Modified Process Films

\begin{tabular}{ll}
\hline $\begin{array}{l}\text { TFN } \\
\text { Number }\end{array}$ & $\begin{array}{l}\text { Average } \\
\text { TCR } \\
\left.\text { (ppm/ } /{ }^{\circ} \mathrm{C}\right)\end{array}$ \\
\hline 031 & -106.5 \\
032 & -104.4 \\
033 & -107.2 \\
076 & -103.5 \\
077 & -103.0 \\
078 & -108.4 \\
103 & -105.7 \\
104 & -103.6 \\
105 & -105.6 \\
112 & -105.7 \\
113 & -104.1 \\
114 & -108.0 \\
121 & -105.2 \\
122 & -104.4 \\
123 & -107.6 \\
\hline
\end{tabular}


Table 5. Life Stability of Standard Process Films

\begin{tabular}{|c|c|}
\hline $\begin{array}{l}\text { TFN } \\
\text { Number }\end{array}$ & $\begin{array}{l}\text { Percent } \\
\text { Deviation* }\end{array}$ \\
\hline $\begin{array}{l}040 \\
041 \\
042 \\
085 \\
086\end{array}$ & $\begin{array}{l}0.18 \\
0.14 \\
0.13 \\
0.13 \\
0.11\end{array}$ \\
\hline $\begin{array}{l}087 \\
130 \\
131 \\
132\end{array}$ & $\begin{array}{l}0.12 \\
0.14 \\
0.14 \\
0.14\end{array}$ \\
\hline \multicolumn{2}{|c|}{$\begin{array}{l}\text { *Percent change after } 200 \text { hours } \\
\text { at } 150^{\circ} \mathrm{C} \text {. }\end{array}$} \\
\hline
\end{tabular}

Table 6. Life Stability of Modified Process Films

\begin{tabular}{|c|c|}
\hline $\begin{array}{l}\text { TFN } \\
\text { Number }\end{array}$ & $\begin{array}{l}\text { Percent } \\
\text { Deviation }\end{array}$ \\
\hline $\begin{array}{l}031 \\
032 \\
033 \\
076 \\
077\end{array}$ & $\begin{array}{l}1.22 \\
1.44 \\
1.29 \\
0.07 \\
0.07\end{array}$ \\
\hline $\begin{array}{l}078 \\
103 \\
104 \\
105 \\
112\end{array}$ & $\begin{array}{l}0.06 \\
0.14 \\
0.13 \\
0.14 \\
0.07\end{array}$ \\
\hline $\begin{array}{l}113 \\
114 \\
121 \\
122 \\
123\end{array}$ & $\begin{array}{l}0.03 \\
0.05 \\
0.07 \\
0.06 \\
0.07\end{array}$ \\
\hline \multicolumn{2}{|c|}{$\begin{array}{l}\text { *Percent change after } 200 \text { hours } \\
\text { at } 150^{\circ} \mathrm{C} \text {. }\end{array}$} \\
\hline
\end{tabular}


A lead pull test was used to determine film bondability in this evaluation. This test required bonding gold-plated copper alloy leads to the gold surface and then pulling the leads at right angles to the gold film. In a lead pull test, the lead can break, the bond can fail, or a failure can occur at the ceramic/tantalum nitride or $\mathrm{Ta}_{2} \mathrm{~N}-\mathrm{Cr} / \mathrm{Au}$ interface. Table 7 lists the various failure mode types and their definitions.

\section{Accelerated Aging}

Five TFNs, processed by the modified process, and one TFN, processed by the standard sequence, were baked in air for 4 hours at $200^{\circ} \mathrm{C}$. Gold-plated lead frames were TC bonded to each TFN, and leads were pulled at $90^{\circ}$ to the substrate surface after 0,21 , and 56 days. The pull strengths and failure modes obtained are shown in Table 8. Bondability results for the standard processed films were excellent; however, the modified processed films exhibited a high percentage of bond delaminations, and TFN number 20 had mixed mode metallization failures.

\section{Backside Bondability}

Gold-plated lead frames were TC bonded to the backside metallization of TFNs which were processed using both the standard and the modified process. These results are shown in Table 9. Pull strength results in all cases were excellent with 100 percent heel breaks (Type B).

\section{Frontside "J" Circuit Bondability}

Table 10 shows the lead frame bondability results of four TFNs which were photoprocessed with a "J" circuit pattern. Goldplated lead frames were bonded to the substrates and the leads were pulled. Two samples had excellent pull strengths with 100 percent heel breaks, while the other two samples had a high percentage of bond delaminations.

Age-Bond-Pull

The age-bond-pull test consisted of 15 TFNs processed by the modified process and 5 TFNs processed by the standard sequence for control. One lead frame was bonded to each substrate as quickly as possible after photoprocessing and then pulled. These results are shown for the time of 0 days in Table 11 . After both 21 and 56 days, another lead frame was bonded to a different side of the same TFN, the leads were pulled, and the failure modes were recorded. The resulsts show an increased percentage of bond delaminations (Type $C$ failures) with aging. Surface contamination could have been a problem since the TFNs were not cleaned between successive bonding operations. There were also two TFNs 115 and 117, which exhibited metallization failures. 
Table 7. Failure Mode Definitions for Lead Frame Bonding

\begin{tabular}{|c|c|c|c|}
\hline \multicolumn{2}{|c|}{$\begin{array}{l}\text { Failure } \\
\text { Mode }\end{array}$} & \multirow{2}{*}{$\begin{array}{l}\text { Failure } \\
\text { Mechanism } \\
\text { Lead Tensile } \\
\text { Failure }\end{array}$} & \multirow{2}{*}{$\begin{array}{l}\text { Definition } \\
\text { Lead breaks at a point other than } \\
\text { in or near the bond zone. }\end{array}$} \\
\hline Type & $\mathbf{A}$ & & \\
\hline Type & B & Heel Failure & $\begin{array}{l}\text { Lead breaks in or near the bond } \\
\text { zone; }>25 \text { percent of bond } \\
\text { deformation area remains. }\end{array}$ \\
\hline Type & C & $\begin{array}{l}\text { Bond } \\
\text { Delamination }\end{array}$ & $\begin{array}{l}\text { Lead peels away from substrate } \\
\text { at the lead frame gold to } \\
\text { substrate interface; lead and } \\
\text { bonding pad visually covered with } \\
\text { gold over } 70 \text { percent of bond zone. }\end{array}$ \\
\hline Type & $E$ & $\begin{array}{l}\text { Substrate-Ta } a_{2} \mathrm{~N} \\
\text { Interface }\end{array}$ & $\begin{array}{l}\text { Lead peels away from substrate; } \\
\text { ceramic is visible over more than } \\
30 \text { percent of the bond zone. }\end{array}$ \\
\hline Type & $\mathbf{F}$ & $\begin{array}{l}\text { Lead Plating } \\
\text { Failure }\end{array}$ & $\begin{array}{l}\text { Lead peels away from substrate; } \\
\text { base copper material is exposed } \\
\text { in the bond zones, on the lead, } \\
\text { or the underlying nickel plating } \\
\text { on the lead is exposed. }\end{array}$ \\
\hline Type & $\mathbf{M}$ & $\begin{array}{l}\text { Metallization } \\
\text { Failure }\end{array}$ & $\begin{array}{l}\text { Lead breaks or peels away from the } \\
\text { substrate with tantalum or chromium } \\
\text { metallization visible over more } \\
\text { than } 10 \text { percent of the exposed } \\
\text { portion of the bond imprint. }\end{array}$ \\
\hline
\end{tabular}

Bond-Age-Pull

The bond-age-pull test consisted of 14 TFNs processed by the modified process and six control samples processed by the standard sequence. Gold-plated lead frames were bonded to three sides of each substrate after photoprocessing. Leads were pulled on one side of each substrate at 0,56 , and 70 days, and pull strengths and failure modes were recorded. Results from this test were both erratic and inconsistent... There were a large number of bond delaminations (Type C failures)" but there were no evidences of any metallization failures. All of the bond-age-pull results are shown in Table 12. 
Table 8. Accelerated Aging ( 4 Hours at $200^{\circ} \mathrm{C}$ ) Versus Thermocompression Bondability for Standard and Modified Process Films

\begin{tabular}{|c|c|c|c|c|c|c|c|c|c|}
\hline \multirow{2}{*}{$\begin{array}{l}\text { TFN } \\
\text { Number }\end{array}$} & \multirow{2}{*}{$\begin{array}{l}\text { Process } \\
\text { Type }\end{array}$} & \multirow{2}{*}{$\begin{array}{l}\text { Leads } \\
\text { Pulled }\end{array}$} & \multirow{2}{*}{$\begin{array}{l}\text { Time } \\
\text { (Days) }\end{array}$} & \multicolumn{3}{|c|}{ Pull Strength (N) } & \multicolumn{3}{|c|}{$\begin{array}{l}\text { Failures } \\
\text { (Percent) }\end{array}$} \\
\hline & & & & $\overline{\mathrm{X}}$ & Minimum & Maximum & $\mathrm{B}$ & $\mathrm{C}$ & $\mathbf{M}$ \\
\hline $\begin{array}{cc}\vdots & \vdots \\
\vdots & \vdots \\
\vdots & \vdots \\
\vdots & \end{array}$ & Standard & $\begin{array}{l}18 \\
18 \\
20\end{array}$ & $\begin{array}{r}0 \\
21 \\
56\end{array}$ & $\begin{array}{l}13.4 \\
11.1 \\
13.4\end{array}$ & $\begin{array}{r}11.1 \\
8.0 \\
9.3\end{array}$ & $\begin{array}{l}15.6 \\
13.4 \\
16.0\end{array}$ & $\begin{array}{r}100 \\
94 \\
95\end{array}$ & $\begin{array}{l}0 \\
6 \\
5\end{array}$ & $\begin{array}{l}0 \\
0 \\
0\end{array}$ \\
\hline$\vdots$ & Modified & $\begin{array}{l}18 \\
18 \\
20\end{array}$ & $\begin{array}{r}0 \\
21 \\
56\end{array}$ & $\begin{array}{r}11.8 \\
8.1 \\
12.0\end{array}$ & $\begin{array}{l}8.9 \\
4.0 \\
9.3\end{array}$ & $\begin{array}{l}13.8 \\
14.2 \\
15.6\end{array}$ & $\begin{array}{r}100 \\
33 \\
75\end{array}$ & $\begin{array}{r}0 \\
50 \\
20\end{array}$ & $\begin{array}{r}0 \\
17 \\
5\end{array}$ \\
\hline 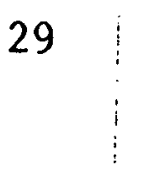 & Modified & $\begin{array}{l}18 \\
18 \\
20\end{array}$ & $\begin{array}{r}0 \\
21 \\
56\end{array}$ & $\begin{array}{l}11.5 \\
11.1 \\
10.6\end{array}$ & $\begin{array}{l}7.6 \\
5.8 \\
4.0\end{array}$ & $\begin{array}{l}14.2 \\
14.7 \\
14.2\end{array}$ & $\begin{array}{l}78 \\
61 \\
95\end{array}$ & $\begin{array}{r}22 \\
39 \\
5\end{array}$ & $\begin{array}{l}0 \\
0 \\
0\end{array}$ \\
\hline $\begin{array}{c}\vdots \\
\vdots \\
\vdots \\
\vdots\end{array}$ & Modified & $\begin{array}{l}18 \\
18 \\
20\end{array}$ & $\begin{array}{r}0 \\
21 \\
56\end{array}$ & $\begin{array}{r}9.7 \\
9.3 \\
10.8\end{array}$ & $\begin{array}{l}4.0 \\
4.5 \\
7.1\end{array}$ & $\begin{array}{l}13.8 \\
12.9 \\
13.8\end{array}$ & $\begin{array}{l}78 \\
44 \\
90\end{array}$ & $\begin{array}{l}22 \\
56 \\
10\end{array}$ & $\begin{array}{l}0 \\
0 \\
0\end{array}$ \\
\hline 110 & Modified & $\begin{array}{l}18 \\
18 \\
20\end{array}$ & $\begin{array}{r}0 \\
21 \\
56\end{array}$ & $\begin{array}{r}10.2 \\
6.5 \\
10.0\end{array}$ & $\begin{array}{l}5.8 \\
0.9 \\
4.5\end{array}$ & $\begin{array}{l}13.4 \\
12.9 \\
14.2\end{array}$ & $\begin{array}{l}50 \\
17 \\
56\end{array}$ & $\begin{array}{l}50 \\
83 \\
44\end{array}$ & $\begin{array}{l}0 \\
0 \\
0\end{array}$ \\
\hline 119 & Modified & $\begin{array}{l}18 \\
18 \\
20\end{array}$ & $\begin{array}{r}0 \\
21 \\
56\end{array}$ & $\begin{array}{r}9.7 \\
5.6 \\
10.0\end{array}$ & $\begin{array}{l}4.5 \\
2.7 \\
4.9\end{array}$ & $\begin{array}{r}12.0 \\
8.5 \\
13.8\end{array}$ & $\begin{array}{r}56 \\
0 \\
50\end{array}$ & $\begin{array}{r}44 \\
100 \\
50\end{array}$ & $\begin{array}{l}0 \\
0 \\
0\end{array}$ \\
\hline
\end{tabular}

*There were no Type A, E, or F failures. 
Table 9. Thermocompression Bondability of Back Side Metallization

\begin{tabular}{|c|c|c|c|c|}
\hline \multirow{2}{*}{$\begin{array}{l}\text { TFN } \\
\text { Number }\end{array}$} & \multirow{2}{*}{$\begin{array}{l}\text { Process } \\
\text { Type }\end{array}$} & \multicolumn{3}{|c|}{ Pull Strength* (N) } \\
\hline & & $\overline{\mathrm{x}}$ & Minimum & Maximum \\
\hline 30 & Modified & 15.9 & 14.2 & 16.9 \\
\hline 66 & Modified & 15.8 & 11.1 & 17.4 \\
\hline 111 & Modified & 15.3 & 13.4 & 16.5 \\
\hline 84 & Standard & 13.7 & 11.6 & 15.1 \\
\hline
\end{tabular}

Table 10. Thermocompression Bondability of Front Side "J" Circuits, Modified Process

\begin{tabular}{|c|c|c|c|c|c|}
\hline \multirow[b]{2}{*}{$\begin{array}{l}\text { TFN } \\
\text { Number }\end{array}$} & \multicolumn{3}{|c|}{ Pull Strength* (N) } & \multicolumn{2}{|c|}{$\begin{array}{l}\text { Failurest } \\
\text { (Percent) }\end{array}$} \\
\hline & $\overline{\mathrm{x}}$ & Minimum & Maximum & B & C \\
\hline 05 & 9.0 & 3.1 & 12.5 & 35 & 65 \\
\hline 48 & 13.6 & 11.6 & 14.7 & 100 & \\
\hline 50 & 13.2 & 11.1 & 14.2 & 100 & \\
\hline 93 & 11.2 & 8.0 & 14.7 & 60 & 40 \\
\hline
\end{tabular}

tOn each TFN, 20 leads were pulled. $\star *$ There were no Type A, E, F, or M failures.

Via Resistance Testing

Thin film hybrid microcircuits require metallized vias (throughholes) to interconnect frontside metallization with metallized 
Table 11. Age-Bond-Pull Results for Standard and Modified Process Films

\begin{tabular}{|c|c|c|c|c|c|c|c|c|c|}
\hline \multirow{2}{*}{$\begin{array}{l}\text { TFN } \\
\text { Number }\end{array}$} & \multirow{2}{*}{$\begin{array}{l}\text { Process } \\
\text { Type }\end{array}$} & \multirow{2}{*}{$\begin{array}{l}\text { Leads } \\
\text { Pulled }\end{array}$} & \multirow{2}{*}{$\begin{array}{l}\text { Time } \\
\text { (Days) }\end{array}$} & \multicolumn{3}{|c|}{ Pull Strength (N) } & \multicolumn{3}{|c|}{$\begin{array}{l}\text { Failurest } \\
\text { (Percent) }\end{array}$} \\
\hline & & & & $\overline{\mathrm{x}}$ & Minimum & Maximum & B & c & M \\
\hline 25 & Modified & $\begin{array}{l}19 \\
19 \\
19\end{array}$ & $\begin{array}{r}0 \\
21 \\
56\end{array}$ & $\begin{array}{l}12.1 \\
10.8 \\
10.1\end{array}$ & $\begin{array}{l}8.9 \\
6.7 \\
5.8\end{array}$ & $\begin{array}{l}14.7 \\
13.4 \\
13.4\end{array}$ & $\begin{array}{r}89 \\
58 \\
63\end{array}$ & $\begin{array}{l}11 \\
42 \\
37\end{array}$ & $\begin{array}{l}0 \\
0 \\
0\end{array}$ \\
\hline 27 & Modified & $\begin{array}{l}19 \\
19 \\
19\end{array}$ & $\begin{array}{r}0 \\
21 \\
56\end{array}$ & $\begin{array}{r}12.6 \\
9.1 \\
11.7\end{array}$ & $\begin{array}{r}10.2 \\
4.5 \\
8.9\end{array}$ & $\begin{array}{l}14.7 \\
12.5 \\
13.4\end{array}$ & $\begin{array}{r}100 \\
37 \\
68\end{array}$ & $\begin{array}{r}0 \\
63 \\
32\end{array}$ & $\begin{array}{l}0 \\
0 \\
0\end{array}$ \\
\hline 34 & Modified & $\begin{array}{l}19 \\
19 \\
19\end{array}$ & $\begin{array}{r}0 \\
21 \\
56\end{array}$ & $\begin{array}{l}12.8 \\
10.8 \\
12.4\end{array}$ & $\begin{array}{r}8.9 \\
8.5 \\
10.7\end{array}$ & $\begin{array}{l}15.6 \\
14.2 \\
13.8\end{array}$ & $\begin{array}{l}84 \\
37 \\
74\end{array}$ & $\begin{array}{l}16 \\
63 \\
26\end{array}$ & $\begin{array}{l}0 \\
0 \\
0\end{array}$ \\
\hline 36 & Modified & $\begin{array}{l}19 \\
19 \\
19\end{array}$ & $\begin{array}{r}0 \\
21 \\
56\end{array}$ & $\begin{array}{l}13.3 \\
11.0 \\
11.6\end{array}$ & $\begin{array}{l}8.5 \\
6.7 \\
8.9\end{array}$ & $\begin{array}{l}15.6 \\
14.2 \\
15.1\end{array}$ & $\begin{array}{l}95 \\
58 \\
58\end{array}$ & $\begin{array}{r}5 \\
42 \\
42\end{array}$ & $\begin{array}{l}0 \\
0 \\
0\end{array}$ \\
\hline 43 & Modified & $\begin{array}{l}19 \\
19 \\
19\end{array}$ & $\begin{array}{r}0 \\
21 \\
56\end{array}$ & $\begin{array}{l}13.7 \\
11.7 \\
11.0\end{array}$ & $\begin{array}{r}10.2 \\
9.8 \\
7.1\end{array}$ & $\begin{array}{l}16.0 \\
13.8 \\
12.9\end{array}$ & $\begin{array}{r}100 \\
79 \\
74\end{array}$ & $\begin{array}{r}0 \\
21 \\
26\end{array}$ & $\begin{array}{l}0 \\
0 \\
0\end{array}$ \\
\hline 45 & Standard & $\begin{array}{l}19 \\
19 \\
19\end{array}$ & $\begin{array}{r}0 \\
21 \\
56\end{array}$ & $\begin{array}{r}12.8 \\
8.8 \\
9.5\end{array}$ & $\begin{array}{l}7.6 \\
4.5 \\
5.3\end{array}$ & $\begin{array}{l}15.6 \\
11.1 \\
12.5\end{array}$ & $\begin{array}{l}79 \\
42 \\
47\end{array}$ & $\begin{array}{l}21 \\
58 \\
53\end{array}$ & $\begin{array}{l}0 \\
0 \\
0\end{array}$ \\
\hline 70 & Modified & $\begin{array}{l}19 \\
19 \\
19\end{array}$ & $\begin{array}{r}0 \\
21 \\
56\end{array}$ & $\begin{array}{l}12.5 \\
10.4 \\
10.9\end{array}$ & $\begin{array}{r}11.1 \\
8.0 \\
5.3\end{array}$ & $\begin{array}{l}14.2 \\
12.5 \\
13.8\end{array}$ & $\begin{array}{l}95 \\
58 \\
68\end{array}$ & $\begin{array}{r}5 \\
42 \\
32\end{array}$ & $\begin{array}{l}0 \\
0 \\
0\end{array}$ \\
\hline 72 & Modified & $\begin{array}{l}19 \\
19 \\
19\end{array}$ & $\begin{array}{r}0 \\
21 \\
56\end{array}$ & $\begin{array}{r}12.9 \\
9.5 \\
13.5\end{array}$ & $\begin{array}{r}11.6 \\
7.1 \\
11.6\end{array}$ & $\begin{array}{l}13.8 \\
12.0 \\
15.6\end{array}$ & $\begin{array}{r}100 \\
42 \\
95\end{array}$ & $\begin{array}{r}0 \\
58 \\
5\end{array}$ & $\begin{array}{l}0 \\
0 \\
0\end{array}$ \\
\hline 79 & Modified & $\begin{array}{l}19 \\
19 \\
19\end{array}$ & $\begin{array}{r}0 \\
21 \\
56\end{array}$ & $\begin{array}{r}13.2 \\
11.5 \\
8.7\end{array}$ & $\begin{array}{l}3.6 \\
5.3 \\
6.2\end{array}$ & $\begin{array}{l}15.1 \\
14.2 \\
11.6\end{array}$ & $\begin{array}{l}95 \\
63 \\
21\end{array}$ & $\begin{array}{r}5 \\
37 \\
79\end{array}$ & $\begin{array}{l}0 \\
0 \\
0\end{array}$ \\
\hline
\end{tabular}


Table 11 Continued. Age-Bond-Pull Results for Standard and Modified Process Films

\begin{tabular}{|c|c|c|c|c|c|c|c|c|c|}
\hline \multirow{2}{*}{$\begin{array}{l}\text { TFN } \\
\text { Number }\end{array}$} & \multirow{2}{*}{$\begin{array}{l}\text { Process } \\
\text { Type }\end{array}$} & \multirow{2}{*}{$\begin{array}{l}\text { Leads } \\
\text { Pulled }\end{array}$} & \multirow{2}{*}{$\begin{array}{l}\text { Time } \\
\text { (Days) }\end{array}$} & \multicolumn{2}{|c|}{ Pull Strength (N) } & \multirow{2}{*}{$\overline{\text { Maximum }}$} & \multicolumn{3}{|c|}{$\begin{array}{l}\text { Failurest } \\
\text { (Percent) }\end{array}$} \\
\hline & & & & $\overline{\mathrm{X}}$ & Minimum & & B & $\mathrm{C}$ & M \\
\hline 81 & Standard & $\begin{array}{l}19 \\
19 \\
19\end{array}$ & $\begin{array}{r}0 \\
21 \\
56\end{array}$ & $\begin{array}{l}12 \cdot 3 \\
10.3 \\
10.3\end{array}$ & $\begin{array}{l}9.8 \\
6.7 \\
6.7\end{array}$ & $\begin{array}{l}14.7 \\
12.5 \\
12.9\end{array}$ & $\begin{array}{l}95 \\
58 \\
47\end{array}$ & $\begin{array}{r}5 \\
42 \\
53\end{array}$ & $\begin{array}{l}0 \\
0 \\
0\end{array}$ \\
\hline 88 & Standard & $\begin{array}{l}19 \\
19 \\
19\end{array}$ & $\begin{array}{r}0 \\
21 \\
56\end{array}$ & $\begin{array}{r}12.8 \\
12.1 \\
8.9\end{array}$ & $\begin{array}{l}6.7 \\
9.8 \\
5.3\end{array}$ & $\begin{array}{l}15.1 \\
13.8 \\
12.4\end{array}$ & $\begin{array}{l}95 \\
84 \\
16\end{array}$ & $\begin{array}{r}5 \\
16 \\
84\end{array}$ & $\begin{array}{l}0 \\
0 \\
0\end{array}$ \\
\hline 90 & Standard & $\begin{array}{l}18 \\
18 \\
19\end{array}$ & $\begin{array}{r}0 \\
21 \\
56\end{array}$ & $\begin{array}{l}10.6 \\
10.5 \\
11.1\end{array}$ & $\begin{array}{l}6.2 \\
7.1 \\
7.1\end{array}$ & $\begin{array}{l}13.4 \\
12.9 \\
12.9\end{array}$ & $\begin{array}{l}67 \\
72 \\
79\end{array}$ & $\begin{array}{l}33 \\
28 \\
21\end{array}$ & $\begin{array}{l}0 \\
0 \\
0\end{array}$ \\
\hline 106 & Modified & $\begin{array}{l}19 \\
19 \\
19\end{array}$ & $\begin{array}{r}0 \\
21 \\
56\end{array}$ & $\begin{array}{l}13.4 \\
10.2 \\
10.6\end{array}$ & $\begin{array}{r}11.6 \\
6.7 \\
7.1\end{array}$ & $\begin{array}{l}15.1 \\
13.4 \\
13.8\end{array}$ & $\begin{array}{r}100 \\
53 \\
53\end{array}$ & $\begin{array}{r}0 \\
47 \\
47\end{array}$ & $\begin{array}{l}0 \\
0 \\
0\end{array}$ \\
\hline 108 & Modified & $\begin{array}{l}19 \\
19 \\
19\end{array}$ & $\begin{array}{r}0 \\
21 \\
56\end{array}$ & $\begin{array}{l}12.7 \\
10.4 \\
12.0\end{array}$ & $\begin{array}{r}10.7 \\
6.2 \\
9.3\end{array}$ & $\begin{array}{l}15.6 \\
13.4 \\
13.8\end{array}$ & $\begin{array}{r}100 \\
63 \\
84\end{array}$ & $\begin{array}{r}0 \\
37 \\
16\end{array}$ & $\begin{array}{l}0 \\
0 \\
0\end{array}$ \\
\hline 115 & Modified & $\begin{array}{l}19 \\
19 \\
19\end{array}$ & $\begin{array}{r}0 \\
21 \\
56\end{array}$ & $\begin{array}{r}10.2 \\
6.8 \\
6.1\end{array}$ & $\begin{array}{l}4.9 \\
4.0 \\
4.0\end{array}$ & $\begin{array}{r}13.4 \\
11.1 \\
9.8\end{array}$ & $\begin{array}{r}68 \\
0 \\
5\end{array}$ & $\begin{array}{r}11 \\
100 \\
95\end{array}$ & $\begin{array}{r}21 \\
0 \\
0\end{array}$ \\
\hline 117 & Modified & $\begin{array}{l}19 \\
19 \\
19\end{array}$ & $\begin{array}{r}0 \\
21 \\
56\end{array}$ & $\begin{array}{r}11.0 \\
9.1 \\
9.6\end{array}$ & $\begin{array}{l}7.6 \\
4.9 \\
5.8\end{array}$ & $\begin{array}{l}13.4 \\
12.5 \\
12.0\end{array}$ & $\begin{array}{l}84 \\
37 \\
21\end{array}$ & $\begin{array}{l}11 \\
63 \\
79\end{array}$ & $\begin{array}{l}5 \\
0 \\
0\end{array}$ \\
\hline 124 & Modified & $\begin{array}{l}19 \\
19 \\
19\end{array}$ & $\begin{array}{r}0 \\
21 \\
56\end{array}$ & $\begin{array}{r}11.3 \\
6.5 \\
10.8\end{array}$ & $\begin{array}{l}8.0 \\
4.0 \\
5.8\end{array}$ & $\begin{array}{l}13.4 \\
12.5 \\
13.4\end{array}$ & $\begin{array}{r}56 \\
5 \\
42\end{array}$ & $\begin{array}{l}44 \\
95 \\
58\end{array}$ & $\begin{array}{l}0 \\
0 \\
0\end{array}$ \\
\hline 126 & Modified & $\begin{array}{l}18 \\
17 \\
19\end{array}$ & $\begin{array}{r}0 \\
21 \\
56\end{array}$ & $\begin{array}{r}9.1 \\
11.3 \\
10.1\end{array}$ & $\begin{array}{l}5.8 \\
8.0 \\
5.8\end{array}$ & $\begin{array}{l}12.0 \\
14.2 \\
14.2\end{array}$ & $\begin{array}{l}39 \\
59 \\
21\end{array}$ & $\begin{array}{l}61 \\
41 \\
78\end{array}$ & $\begin{array}{l}0 \\
0 \\
0\end{array}$ \\
\hline
\end{tabular}


Table 11 Continued. Age-Bond-Pull Results for Standard and Modified Process Films

\begin{tabular}{|c|c|c|c|c|c|c|c|c|c|}
\hline \multirow{2}{*}{$\begin{array}{l}\text { TFN } \\
\text { Number }\end{array}$} & \multirow{2}{*}{$\begin{array}{l}\text { Process } \\
\text { Type }\end{array}$} & \multirow{2}{*}{$\begin{array}{l}\text { Leads } \\
\text { Pulled }\end{array}$} & \multirow{2}{*}{$\begin{array}{l}\text { Time } \\
\text { (Days) }\end{array}$} & \multicolumn{3}{|c|}{ Pull Strength (N) } & \multicolumn{3}{|c|}{$\begin{array}{l}\text { Failurest } \\
\text { (Percent) }\end{array}$} \\
\hline & & & & $\overline{\mathrm{X}}$ & Minimum & Maximum & B & $\mathrm{C}$ & $\mathbf{M}$ \\
\hline 133 & Standard & $\begin{array}{l}19 \\
19 \\
19\end{array}$ & $\begin{array}{r}0 \\
21 \\
56\end{array}$ & $\begin{array}{l}12.1 \\
10.5 \\
11.5\end{array}$ & $\begin{array}{r}10.2 \\
8.5 \\
8.0\end{array}$ & $\begin{array}{l}14.7 \\
11.6 \\
13.8\end{array}$ & $\begin{array}{r}100 \\
95 \\
53\end{array}$ & $\begin{array}{r}0 \\
5 \\
47\end{array}$ & $\begin{array}{l}0 \\
0 \\
0\end{array}$ \\
\hline 135 & Standard & $\begin{array}{l}19 \\
19 \\
19\end{array}$ & $\begin{array}{r}0 \\
21 \\
56\end{array}$ & $\begin{array}{l}12.0 \\
12.4 \\
10.9\end{array}$ & $\begin{array}{r}7.6 \\
11.1 \\
7.6\end{array}$ & $\begin{array}{l}13.8 \\
13.8 \\
13.4\end{array}$ & $\begin{array}{l}95 \\
95 \\
53\end{array}$ & $\begin{array}{r}5 \\
5 \\
47\end{array}$ & $\begin{array}{l}0 \\
0 \\
0\end{array}$ \\
\hline
\end{tabular}

*There were no Type A, E, or F failures. 
Table 12. Bond-Age-Pull Results for Standard and Modified Process Films

\begin{tabular}{|c|c|c|c|c|c|c|c|c|c|}
\hline \multirow{2}{*}{$\begin{array}{l}\text { TFN } \\
\text { Number }\end{array}$} & & \multirow{2}{*}{$\begin{array}{l}\text { Process } \\
\text { Type }\end{array}$} & \multirow{2}{*}{$\begin{array}{l}\text { Leads } \\
\text { Pulled }\end{array}$} & \multirow{2}{*}{$\begin{array}{l}\text { Time } \\
\text { (Days). }\end{array}$} & \multicolumn{2}{|c|}{ Pull Strength (N) } & \multirow{2}{*}{ Maximum } & \multicolumn{2}{|c|}{$\begin{array}{l}\text { Failures* } \\
\text { (Percent) }\end{array}$} \\
\hline & & & & & $\overline{\mathrm{X}}$ & Minimum & & B & C \\
\hline 19 & 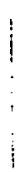 & Modified & $\begin{array}{l}18 \\
18 \\
20\end{array}$ & $\begin{array}{r}0 \\
56 \\
70\end{array}$ & $\begin{array}{r}12.3 \\
8.3 \\
10.9\end{array}$ & $\begin{array}{l}9.8 \\
3.6 \\
5.8\end{array}$ & $\begin{array}{l}14.2 \\
13.4 \\
13.8\end{array}$ & $\begin{array}{l}83 \\
28 \\
85\end{array}$ & $\begin{array}{l}17 \\
72 \\
15\end{array}$ \\
\hline 26 & & Modified & $\begin{array}{l}18 \\
18 \\
20\end{array}$ & $\begin{array}{r}0 \\
56 \\
70\end{array}$ & $\begin{array}{r}10.1 \\
8.6 \\
9.2\end{array}$ & $\begin{array}{l}5.8 \\
6.2 \\
5.8\end{array}$ & $\begin{array}{l}14.2 \\
10.7 \\
11.6\end{array}$ & $\begin{array}{l}22 \\
17 \\
40\end{array}$ & $\begin{array}{l}78 \\
83 \\
60\end{array}$ \\
\hline 28 & & Modified & $\begin{array}{l}18 \\
18 \\
20\end{array}$ & $\begin{array}{r}0 \\
56 \\
70\end{array}$ & $\begin{array}{l}12.4 \\
11.9 \\
12.1\end{array}$ & $\begin{array}{l}9.3 \\
7.1 \\
7.1\end{array}$ & $\begin{array}{l}14.7 \\
13.8 \\
13.4\end{array}$ & $\begin{array}{l}61 \\
56 \\
75\end{array}$ & $\begin{array}{l}39 \\
44 \\
25\end{array}$ \\
\hline 35 & & Modified & $\begin{array}{l}18 \\
18 \\
20\end{array}$ & $\begin{array}{r}0 \\
56 \\
70\end{array}$ & $\begin{array}{r}11.6 \\
9.0 \\
10.7\end{array}$ & $\begin{array}{l}6.7 \\
5.8 \\
4.9\end{array}$ & $\begin{array}{l}14.7 \\
11.1 \\
14.2\end{array}$ & $\begin{array}{r}61 \\
0 \\
70\end{array}$ & $\begin{array}{r}39 \\
100 \\
30\end{array}$ \\
\hline 37 & & Standard & $\begin{array}{l}18 \\
18 \\
20\end{array}$ & $\begin{array}{r}0 \\
56 \\
70\end{array}$ & $\begin{array}{r}11.3 \\
8.3 \\
9.9\end{array}$ & $\begin{array}{r}10.2 \\
3.1 \\
5.8\end{array}$ & $\begin{array}{l}14.2 \\
12.5 \\
12.5\end{array}$ & $\begin{array}{l}44 \\
39 \\
50\end{array}$ & $\begin{array}{l}56 \\
61 \\
50\end{array}$ \\
\hline 44 & & Standard & $\begin{array}{l}18 \\
18 \\
20\end{array}$ & $\begin{array}{r}0 \\
56 \\
70\end{array}$ & $\begin{array}{l}11.0 \\
12.3 \\
10.8\end{array}$ & $\begin{array}{l}5.3 \\
9.8 \\
5.8\end{array}$ & $\begin{array}{l}13.4 \\
13.8 \\
13.8\end{array}$ & $\begin{array}{r}67 \\
100 \\
60\end{array}$ & $\begin{array}{r}33 \\
0 \\
40\end{array}$ \\
\hline 64 & & Modified & $\begin{array}{l}18 \\
18 \\
20\end{array}$ & $\begin{array}{r}0 \\
56 \\
70\end{array}$ & $\begin{array}{l}12.8 \\
10.2 \\
12.1\end{array}$ & $\begin{array}{l}5.3 \\
5.8 \\
8.9\end{array}$ & $\begin{array}{l}15.1 \\
13.8 \\
14.7\end{array}$ & $\begin{array}{l}89 \\
22 \\
65\end{array}$ & $\begin{array}{l}11 \\
78 \\
35\end{array}$ \\
\hline 71 & & Modified & $\begin{array}{l}18 \\
18 \\
20\end{array}$ & $\begin{array}{r}0 \\
56 \\
70\end{array}$ & $\begin{array}{l}9.7 \\
7.5 \\
7.7\end{array}$ & $\begin{array}{l}5.3 \\
5.8 \\
3.1\end{array}$ & $\begin{array}{r}13.4 \\
9.8 \\
12.9\end{array}$ & $\begin{array}{r}56 \\
6 \\
40\end{array}$ & $\begin{array}{l}44 \\
94 \\
60\end{array}$ \\
\hline 73 & : & Modified & $\begin{array}{l}18 \\
18 \\
20\end{array}$ & $\begin{array}{r}0 \\
56 \\
70\end{array}$ & $\begin{array}{r}9.9 \\
10.4 \\
8.8\end{array}$ & $\begin{array}{l}5.3 \\
5.8 \\
2.7\end{array}$ & $\begin{array}{l}14.7 \\
12.5 \\
14.7\end{array}$ & $\begin{array}{l}56 \\
72 \\
50\end{array}$ & $\begin{array}{l}44 \\
28 \\
50\end{array}$ \\
\hline
\end{tabular}


Table 12 Continued. Bond-Age-Pull Results for Standard and Modified Process Films

\begin{tabular}{|c|c|c|c|c|c|c|c|c|c|}
\hline \multirow{2}{*}{\multicolumn{2}{|c|}{$\begin{array}{l}\text { TFN } \\
\text { Number }\end{array}$}} & \multirow{2}{*}{$\begin{array}{l}\text { Process } \\
\text { Type }\end{array}$} & \multirow{2}{*}{$\begin{array}{l}\text { Leads } \\
\text { Pulled }\end{array}$} & \multirow{2}{*}{$\begin{array}{l}\text { Time } \\
\text { (Days) }\end{array}$} & \multicolumn{3}{|c|}{ Pull Strength (N). } & \multicolumn{2}{|c|}{$\begin{array}{l}\text { Failures } \\
\text { (Percent) }\end{array}$} \\
\hline & & & & & $\overline{\mathrm{X}}$ & Minimum & Maximum & B & C \\
\hline 80 & $\vdots$ & Modified & $\begin{array}{l}18 \\
18 \\
20\end{array}$ & $\begin{array}{r}0 \\
56 \\
70\end{array}$ & $\begin{array}{r}10.8 \\
10.8 \\
9.4\end{array}$ & $\begin{array}{l}4.0 \\
7.6 \\
2.2\end{array}$ & $\begin{array}{l}15.1 \\
13.4 \\
14.7\end{array}$ & $\begin{array}{l}50 \\
50 \\
50\end{array}$ & $\begin{array}{l}50 \\
50 \\
50\end{array}$ \\
\hline 82 & 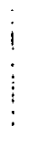 & Standard & $\begin{array}{l}18 \\
18 \\
20\end{array}$ & $\begin{array}{r}0 \\
56 \\
70\end{array}$ & $\begin{array}{l}12.4 \\
12.5 \\
12.0\end{array}$ & $\begin{array}{l}7.6 \\
7.1 \\
7.6\end{array}$ & $\begin{array}{l}14.7 \\
14.7 \\
15.1\end{array}$ & $\begin{array}{l}89 \\
89 \\
70\end{array}$ & $\begin{array}{l}11 \\
11 \\
30\end{array}$ \\
\hline 89 & $\begin{array}{l}: \\
: \\
:\end{array}$ & Standard & $\begin{array}{r}18 \\
9 \\
20\end{array}$ & $\begin{array}{r}0 \\
56 \\
70\end{array}$ & $\begin{array}{l}12.7 \\
10.3 \\
11.2\end{array}$ & $\begin{array}{l}8.9 \\
5.8 \\
5.8\end{array}$ & $\begin{array}{l}14.7 \\
13.4 \\
13.8\end{array}$ & $\begin{array}{l}83 \\
78 \\
75\end{array}$ & $\begin{array}{l}17 \\
22 \\
25\end{array}$ \\
\hline 100 & $\vdots$ & Modified & $\begin{array}{l}18 \\
18 \\
20\end{array}$ & $\begin{array}{r}0 \\
56 \\
70\end{array}$ & $\begin{array}{r}11.3 \\
7.7 \\
10.2\end{array}$ & $\begin{array}{l}5.8 \\
4.0 \\
4.9\end{array}$ & $\begin{array}{r}14.2 \\
9.3 \\
12.9\end{array}$ & $\begin{array}{r}61 \\
6 \\
65\end{array}$ & $\begin{array}{l}39 \\
94 \\
35\end{array}$ \\
\hline 107 & $\vdots$ & Modified & $\begin{array}{l}18 \\
18 \\
20\end{array}$ & $\begin{array}{r}0 \\
56 \\
70\end{array}$ & $\begin{array}{l}12.0 \\
10.5 \\
10.4\end{array}$ & $\begin{array}{l}8.5 \\
7.1 \\
5.8\end{array}$ & $\begin{array}{l}14.7 \\
13.4 \\
14.7\end{array}$ & $\begin{array}{l}83 \\
78 \\
60\end{array}$ & $\begin{array}{l}17 \\
22 \\
40\end{array}$ \\
\hline 109 & & Modified & $\begin{array}{l}18 \\
18 \\
20\end{array}$ & $\begin{array}{r}0 \\
56 \\
70\end{array}$ & $\begin{array}{r}11.7 \\
5.7 \\
10.3\end{array}$ & $\begin{array}{l}4.5 \\
3.1 \\
6.2\end{array}$ & $\begin{array}{l}14.7 \\
11.1 \\
14.7\end{array}$ & $\begin{array}{r}67 \\
0 \\
50\end{array}$ & $\begin{array}{r}33 \\
100 \\
50\end{array}$ \\
\hline 116 & $\vdots$ & Modified & $\begin{array}{l}18 \\
18 \\
20\end{array}$ & $\begin{array}{r}0 \\
56 \\
70\end{array}$ & $\begin{array}{r}10.4 \\
7.5 \\
8.5\end{array}$ & $\begin{array}{l}4.9 \\
3.6 \\
2.7\end{array}$ & $\begin{array}{l}13.8 \\
11.1 \\
12.9\end{array}$ & $\begin{array}{r}50 \\
6 \\
40\end{array}$ & $\begin{array}{l}50 \\
94 \\
60\end{array}$ \\
\hline 118 & 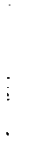 & Modified & $\begin{array}{l}18 \\
18 \\
20\end{array}$ & $\begin{array}{r}0 \\
56 \\
70\end{array}$ & $\begin{array}{r}11.1 \\
7.2 \\
9.3\end{array}$ & $\begin{array}{l}6.7 \\
4.5 \\
4.0\end{array}$ & $\begin{array}{r}13.4 \\
9.8 \\
12.9\end{array}$ & $\begin{array}{r}61 \\
0 \\
35\end{array}$ & $\begin{array}{r}39 \\
100 \\
65\end{array}$ \\
\hline 125 & & Modified & $\begin{array}{l}18 \\
18 \\
20\end{array}$ & $\begin{array}{r}0 \\
56 \\
70\end{array}$ & $\begin{array}{l}9.9 \\
6.0 \\
8.4\end{array}$ & $\begin{array}{l}6.2 \\
3.1 \\
3.6\end{array}$ & $\begin{array}{r}13.8 \\
8.5 \\
12.5\end{array}$ & $\begin{array}{r}39 \\
0 \\
35\end{array}$ & $\begin{array}{r}61 \\
100 \\
65\end{array}$ \\
\hline
\end{tabular}


Table 12 Continued. Bond-Age-Pull Results for Standard and Modified Process Films

\begin{tabular}{|c|c|c|c|c|c|c|c|c|}
\hline \multirow{2}{*}{$\begin{array}{l}\text { TFN } \\
\text { Number }\end{array}$} & \multirow{2}{*}{$\begin{array}{l}\text { Process } \\
\text { Type }\end{array}$} & \multirow{2}{*}{$\begin{array}{l}\text { Leads } \\
\text { Pulled }\end{array}$} & \multirow{2}{*}{$\begin{array}{l}\text { Time } \\
\text { (Days) }\end{array}$} & \multicolumn{3}{|c|}{ Pull Strength (N) } & \multicolumn{2}{|c|}{$\begin{array}{l}\text { Failures } \\
\text { (Percent) }\end{array}$} \\
\hline & & & & $\overline{\mathbf{X}}$ & Minimum & Maximum & B & $\mathrm{C}$ \\
\hline 127 & Standard & $\begin{array}{l}18 \\
18 \\
20\end{array}$ & $\begin{array}{r}0 \\
56 \\
70\end{array}$ & $\begin{array}{l}12.0 \\
13.4 \\
12.1\end{array}$ & $\begin{array}{r}8.5 \\
11.1 \\
8.0\end{array}$ & $\begin{array}{l}15.1 \\
14.7 \\
14.7\end{array}$ & $\begin{array}{l}72 \\
89 \\
85\end{array}$ & $\begin{array}{l}28 \\
11 \\
15\end{array}$ \\
\hline 134 & Standard & $\begin{array}{l}18 \\
18 \\
20\end{array}$ & $\begin{array}{r}0 \\
56 \\
70\end{array}$ & $\begin{array}{l}10.6 \\
10.4 \\
10.4\end{array}$ & $\begin{array}{l}3.1 \\
4.9 \\
4.5\end{array}$ & $\begin{array}{l}13.8 \\
13.8 \\
13.4\end{array}$ & $\begin{array}{l}67 \\
56 \\
50\end{array}$ & $\begin{array}{l}33 \\
44 \\
50\end{array}$ \\
\hline
\end{tabular}

*There were no Type $A, E, F$, or M failures. 
backside ground planes. Via resistance was measured for five vias on each of 19 TFNs processed using the modified sequence. In all cases, the via resistance was below an established maximum of $10 \mathrm{~m} \Omega$ (Table 13).

\section{Solderability Testing}

Capacitors were soldered to four TFNs using 50-50 Pb-In solder to determine if there were any problems in soldering to substrates which had been processed using the modified sequence. All substrates were visually inspected after soldering to ensure integrity of the solder joint. Next, the TFNs were vacuum baked for 4 hours at $100^{\circ} \mathrm{C}$ and then visually checked. The samples were then subjected to 10 temperature cycles between $-40^{\circ} \mathrm{C}$ and $+125^{\circ} \mathrm{C}$. All capacitors were then subjected to a nondestructive shear test at $6.7 \mathrm{~N}$. Finally the samples were subjected to 40 more temperature cycles between $-40^{\circ} \mathrm{C}$ and $+125^{\circ} \mathrm{C}$ and then visually inspected. In all cases, the solder joints looked good, and it was concluded that there would be no problems in soldering to modified process films.

\section{ACCOMPLISHMENTS}

The modified thin film process was investigated in an effort to find a processing sequence that would minimize or possibly eliminate the $\mathrm{Cr} / \mathrm{Au}-\mathrm{to}-\mathrm{Ta}_{2} \mathrm{~N}$ adhesion problems encountered in thin film network manufacturing processes. Results of this work indicate that the modified process would not offer any significant advantages over the current production process. Disadvantages of the modified thin film processing sequence are:

- $\mathrm{Cr} / \mathrm{Au}-\mathrm{to}-\mathrm{Ta}_{2} \mathrm{~N}$ adhesion failures are not eliminated,

- Additional processing steps are required during evaporation and photolithography, and

- Contact resistance is 2 to 3 times higher for the modified process.

Although results obtained for TCR, life stability, via resistance, bondability, and solderability were similiar for both standard and modified process films, efforts on the modified process were discontinued because of the disadvantages listed above. 
Table 13. Via Resistance of Modified Process Films

\begin{tabular}{|c|c|c|c|c|c|}
\hline $\begin{array}{l}\text { TFN } \\
\text { Number }\end{array}$ & $\begin{array}{l}\text { Via } 1 \\
(\mathrm{~m} \Omega)\end{array}$ & $\begin{array}{l}\text { Via } 2 \\
(\mathrm{~m} \Omega)\end{array}$ & $\begin{array}{l}\text { Via } 3 \\
(\mathrm{~m} \Omega)\end{array}$ & $\begin{array}{l}\text { Via } 4 \\
(\mathrm{~m} \Omega)\end{array}$ & $\begin{array}{l}\text { Via } \\
(\mathrm{m} \Omega)\end{array}$ \\
\hline $\begin{array}{l}01 \\
03 \\
05 \\
07 \\
09\end{array}$ & $\begin{array}{l}5.0 \\
5.3 \\
4.9 \\
3.6 \\
4.0\end{array}$ & $\begin{array}{l}4.5 \\
5.4 \\
4.4 \\
5.1 \\
5.5\end{array}$ & $\begin{array}{l}4.5 \\
4.6 \\
4.5 \\
5.0 \\
5.2\end{array}$ & $\begin{array}{l}4.4 \\
4.9 \\
5.4 \\
5.2 \\
5.6\end{array}$ & $\begin{array}{l}4.7 \\
5.2 \\
5.0 \\
5.3 \\
6.3\end{array}$ \\
\hline $\begin{array}{l}11 \\
13 \\
15 \\
17 \\
46\end{array}$ & $\begin{array}{l}4.3 \\
4.1 \\
5.3 \\
5.6 \\
5.5\end{array}$ & $\begin{array}{l}3.8 \\
4.8 \\
4.8 \\
4.8 \\
7.4\end{array}$ & $\begin{array}{l}4.8 \\
4.2 \\
4.7 \\
5.6 \\
6.3\end{array}$ & $\begin{array}{l}4.9 \\
5.1 \\
5.1 \\
5.8 \\
6.9\end{array}$ & $\begin{array}{l}3.9 \\
4.7 \\
6.0 \\
6.5 \\
5.5\end{array}$ \\
\hline $\begin{array}{l}48 \\
50 \\
52 \\
54 \\
91\end{array}$ & $\begin{array}{l}5.1 \\
4.8 \\
8.3 \\
6.6 \\
3.9\end{array}$ & $\begin{array}{l}4.7 \\
5.2 \\
7.9 \\
6.3 \\
4.0\end{array}$ & $\begin{array}{l}5.1 \\
4.4 \\
6.7 \\
6.8 \\
4.7\end{array}$ & $\begin{array}{l}4.9 \\
4.4 \\
8.1 \\
8.5 \\
4.5\end{array}$ & $\begin{array}{l}4.8 \\
4.3 \\
6.2 \\
7.7 \\
4.4\end{array}$ \\
\hline $\begin{array}{l}93 \\
95 \\
97 \\
99\end{array}$ & $\begin{array}{l}4.5 \\
4.6 \\
5.6 \\
5.7\end{array}$ & $\begin{array}{l}4.5 \\
4.7 \\
5.4 \\
5.5\end{array}$ & $\begin{array}{l}4.9 \\
5.7 \\
5.8 \\
6.5\end{array}$ & $\begin{array}{l}4.8 \\
5.7 \\
5.9 \\
6.4\end{array}$ & $\begin{array}{l}5.2 \\
5.2 \\
5.8 \\
6.4\end{array}$ \\
\hline
\end{tabular}


BDX-613-2409

A MODIFIED THIN FILM PROCESSING SEQUENCE, C. R. Baxter, Topical, June 1980.

A modified thin film processing sequence in which tantalum nitride $\left(\mathrm{Ta}_{2} \mathrm{~N}\right)$ resistors are stabilized prior to chromium/gold ( $\mathrm{Cr} / \mathrm{Au}$ ) evaporation was investigated, and the effects of subsequent processing on unstabilized $\mathrm{Cr} / \mathrm{Au}$ films were determined. Thin films evaporated using the modified process yielded results similar to those of films evaporated using the standard processing techniques. Work on the modified process was discontinued because of some metallization adhesion failures at the $\mathrm{Cr} / \mathrm{Au}-\mathrm{to}-\mathrm{Ta}_{2} \mathrm{~N}$ interface, higher 1 contact resistance, and the additional steps required in processing.

ELECTRICAL: Thin Film Processing

A MODIFIED THIN FILM PROCESSING SEQUENCE, C. R. Baxter, Topical, BDX-613-2409; June 1980.

A modified thin film processing sequence in which tantalum nitride $\left(\mathrm{Ta}_{2} \mathrm{~N}\right)$ resistors are stabilized prior to chromium/gold ( $\mathrm{Cr} / \mathrm{Au}$ ) evaporation was investigated, and the effects of subsequent processing on unstabilized $\mathrm{Cr} / \mathrm{Au}$ films were determined. Thin films evaporated using the modified process yielded results similar to those of films evaporated using the standard processing techniques. Work on the modified process was discontinued because of some metallization adhesion failures at the $\mathrm{Cr} / \mathrm{Au}-\mathrm{to}-\mathrm{Ta}_{2} \mathrm{~N}$ interface, higher contact resistance, and the additional steps required in processing.

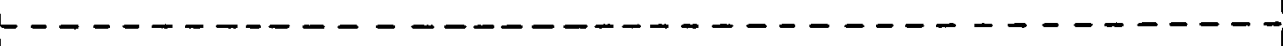

A MODIFIED THIN FILM PROCESSING SEQUENCE, C. R. Baxter, Topical, BDX-613-2409, June 1980.

A modified thin film processing sequence in which tantalum nitride $\left(\mathrm{Ta}_{2} \mathrm{~N}\right)$ resistors are stabilized prior to chromium/gold ( $\mathrm{Cr} / \mathrm{Au}$ ) evaporation was investigated, and the effects of subsequent processing on unstabilized $\mathrm{Cr} / \mathrm{Au}$ films were determined. Thin films evaporated using the modified process yielded results similar to those of films evaporated using the standard processing techiniques. Work on the modified process was discontinued because of some metallization adhesion failures at the $\mathrm{Cr} / \mathrm{Au}-\mathrm{to}-\mathrm{Ta}_{2} \mathrm{~N}$ interface, higher contact resistance, and the additional steps requiring in processing. 